\title{
Do You Know Your Lines?
}

\author{
Vamsidhar Rachapalli ${ }^{1}$ Pankaj Sharma ${ }^{2}$ Sheo Kumar ${ }^{3}$ Mangerira Chinnappa Uthappa ${ }^{1}$
}

${ }^{1}$ Department of Interventional Radiology and Interventional Oncology, BGS Global Hospital, Bangalore, Karnataka, India

2 Department of Radiodiagnosis, Delhi State Cancer Institute, Dilshad Garden, Delhi, India

${ }^{3}$ Department of Radiodiagnosis, Sanjay Gandhi Post Graduate Institute of Medical Sciences, Lucknow, Uttar Pradesh, India

J Clin Interv Radiol ISVIR 2017;1:161-167.
Address for correspondence Vamsidhar Rachapalli, FRCR, Department of Interventional Radiology and Interventional Oncology, BGS Global Hospital, No 67, Uttarahalli Road, Kengeri, Bangalore 560060, Karnataka, India (e-mail: r_vamsidhar@yahoo.com).

\begin{abstract}
Keywords

- venous

- catheters

- selection

The use of central venous catheters has become ubiquitous in the clinical practice. While a majority of them are easy to insert, many consider it a mundane interventional procedure. However, it is important to ensure that the right catheter is selected for the right patient. In addition, due diligence should be observed during site selection and catheter securement. This article briefly but concisely covers various aspects of venous catheter insertion ranging from catheter selection, patient preparation, choice of skin antisepsis to catheter tip position. This article, however, does not deal with the actual steps involved in the insertion of various catheters.
\end{abstract}

\section{Introduction}

The use of vascular access devices (VAD) has become ubiquitous in medical practice. They are used in patient care both in and out of hospital. Their uses range from administration of medicaments to monitoring. Whereas placement of VAD is considered to be a routine and mundane interventional procedure, their improper use is associated with significant morbidity, the most important being catheter-related infection (CRI) and thrombosis. Most of these can be avoided with meticulous attention to technique and appropriate catheter selection.

As VAD are placed by a wide range of clinicians of different backgrounds, there is no standardization in selection or follow-up. In most centers, interventional radiologists are involved in the placement of VAD, particularly in complex cases. Moreover, imaging is of paramount importance when VAD are placed through occluded neck or thoracic veins. The purpose of this article is to highlight to the reader the common type of VAD in use, their indication, patient preparation, and important aspects pertaining to their placement. However, in this article, the authors do not discuss the insertion techniques in detail for the different types of catheters.

received

January 17, 2017

accepted after revision

June 19, 2017

published online

September 1, 2017

\section{Types of Catheters}

\section{Midline Catheters}

These are short-length catheters, approximately 20 to $25 \mathrm{~cm}$. They are inserted at the elbow crease and their tip should not be advanced beyond the axillary vein. ${ }^{1}$ Hence they are not central venous catheters (CVCs). Given the tip location, continuous infusion of greater than $10 \%$ dextrose solution, vesicants, and corrosive infusates is not recommended.

\section{Peripherally Inserted Central Catheter}

Peripherally inserted central catheter (PICC) acts as an intermediary between short- and long-term catheters. They can be placed at the bed side with the tip in a central location; a wide range of infusates can be given. They can have multiple lumens enabling simultaneous administration of medication. PICC can be open ended in which catheter has an end hole or close ended in which catheter has Groshong side valve. Groshong valve allows fluid injection and blood aspiration but prevents air or blood from entering the catheter. Some PICCs can have either proximal or distal valves that prevent the reflux of blood into the distal tip. PICCS are made of silicone or polyurethane-based material. The newer polyurethane power-injectable PICCs offer multiple advantages. They are associated with a lower infection

Copyright (e) 2017 by Indian Society of Vascular and Interventional Radiology

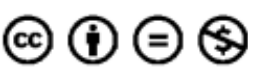


rate when compared with catheters made of polyvinyl chloride or polyethylene. Intravenous contrast can be injected by a pressure pump for undertaking computed tomographic studies. ${ }^{2}$ In addition, polyurethane is a tougher material. Thus the catheter walls are thinner with larger lumens, thereby improving flow rates and reducing catheter breakage. ${ }^{3}$ In some cases, a tunneled PICC can be considered to secure it and reduce the risk of CRI. Recent reviews have shown that there was an increased risk of thrombosis when PICC was used in patients with malignancy and critical illness. ${ }^{4,5}$ Chopra et al have recommended that the risks and benefits should be weighed in such patients prior to the placement of PICCs. ${ }^{5}$ Currently, a randomized control study is underway to ascertain which venous access offers the best outcome in view of safety, clinical effectiveness, and costeffectiveness for cancer patients. ${ }^{6}$

\section{Nontunneled Central Venous Catheter}

Nontunneled central venous catheters (NTCVCs) are indicated for short-term use, and these CVC are generally inserted into the jugular vein. NTCVC insertion is undertaken as a bedside procedure. However, they have high chance of infection and can easily dislodge. NTCVCs are generally placed by clinicians in an acute setting or for emergency dialysis.

\section{Tunneled Central Venous Catheter}

Tunneled central venous catheters (TCVCs) are the mainstay for long-term access. They can have multiple lumens. Discussion with the relevant clinician is recommended to ensure that the appropriate catheter is used. They have lower infection rates than NTCVCs. ${ }^{3}$ TCVCs have a dacron cuff embedded on the shaft. This incites a fibrotic reaction that helps secure the catheter in place and reduce spread of infection.

There are a range of hemodialysis catheters in use. They are generally $14.5 \mathrm{~F}$ dual-lumen devices with lengths varying from 19 to $28 \mathrm{~cm}$ and are capable of handling high flow rates (500-600 $\mathrm{mL} / \mathrm{min}$ ). No single catheter has been shown to be superior to the other. ${ }^{7}$ However, the Palindrome catheter (Bard PV) that has a split and symmetric tip is reported to achieve the lowest recirculation rates among the available

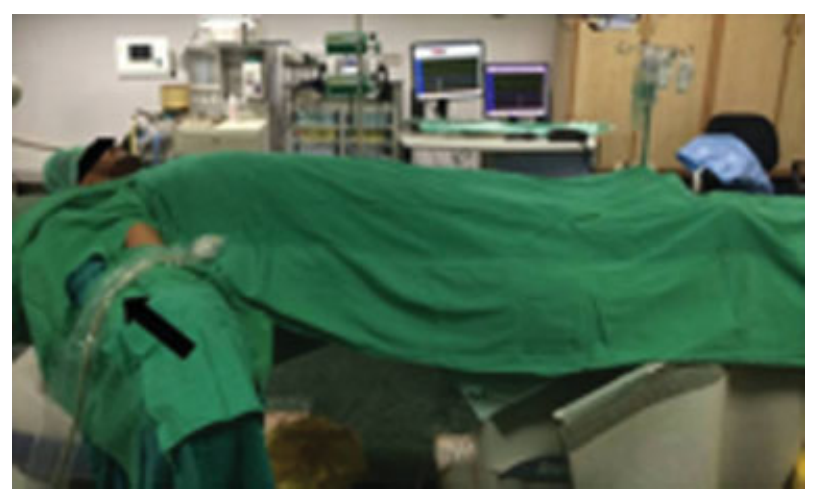

Fig. 1 The patient undergoing a PICC insertion has a full body preparation. The ultrasound probe has been covered with along probe cover. catheters. ${ }^{8}$ Local availability and expertise guide catheter choice. Cuffed catheters are preferred as they have lower infection rates and better flow rates. Noncuffed or temporary catheters should be used in a hospital setting and for a period not longer than 1 week. ${ }^{7}$ Prior to placement of a dialysis catheter, it is important to engage the nephrologists on further treatment plans as fistulas should be the mainstay approach for renal replacement therapy. However, catheters play an important role in emergent situations or when there is a failure of a fistula. As far as possible, the line should not be on the same side as a maturing/proposed fistula site.

Other commonly used tunneled catheters are Hickman (Bard PV) and Broviac (Bard PV) lines. They can have multiple lumens and are routinely used in the administration of chemotherapeutic medications, total parenteral nutrition, and aphaeresis. Unlike the dialysis catheters, catheter length has to be trimmed to the appropriate length prior to insertion.

\section{Implanted Central Venous Access Device}

These are more commonly referred to as port-a-caths or chemoports. These are surgically implanted with no visible catheter. Implanted central venous access devices (ICVADs) are made of titanium and plastic. They have two parts: a silicone or polyurethane catheter and a reservoir. The reservoir is accessed using a special noncoring Huber needle through the skin and the silicone window. They are available as a single- or double-lumen configuration. The port can be placed in the subcutaneous tissue of the chest, arm, thigh, or abdomen. These are best suited for patients requiring longterm treatment such as chemotherapy for oncology patients or antibiotics for cystic fibrosis patients. They are cosmetically acceptable. They have a lower risk of infection when compared with tunneled catheters. ${ }^{3}$

\section{Catheter Selection}

Multitude of factors decide which catheter should be selected. This depends on the nature of the infusate, duration of the therapy, available access points, and further planned therapies/treatments. - Table 1 summarizes the most common catheters in use and the duration they are recommended to be used for.

The use of multiluminal catheters depends on the clinical indication. O'Brien et al showed that by using a single-lumen PICC of the smallest size required, the number of PICCs reinsertions due to infection or thrombosis is reduced. It is particularly important to ensure that infusates are compatible if they are to be infused through a single lumen. ${ }^{9}$ One special consideration is administration of parenteral nutrition. Whereas there is no robust recommendation, it is good practice to dedicate one lumen or place a single-lumen catheter for administration of parenteral nutrition. ${ }^{2}$ Regarding valved PICCs, studies have shown no difference in the occlusion rates between valved and nonvalved PICCs. ${ }^{10,11}$

Antibiotic and anti-infective impregnated catheters and cuffs are available. The common coating agents are chlorhexidine/silver sulfadiazine and minocycline/rifampicin. Though 
Table 1 Type of the common catheters in use with their recommended duration of use

\begin{tabular}{|l|l|l|}
\hline & Peripheral catheter & Central catheter \\
\hline Short term & $\begin{array}{l}\text { IV cannula } \\
\text { (up to 7 d) } \\
\text { Midline catheter } \\
\text { (1-4 wk) }\end{array}$ & $\begin{array}{l}\text { NTVACs } \\
\text { (7-10 d) }\end{array}$ \\
\hline Medium term & Not recommended & PICC lines (1-6 mo) \\
\hline Long term & Not recommended & $\begin{array}{l}\text { TCVC (months to } \\
\text { years) } \\
\text { ICVAD (months to } \\
\text { years) }\end{array}$ \\
\hline
\end{tabular}

Abbreviations: ICVAD, implanted central venous access device; IV, intravenous; NTCVC, nontunneled central venous catheter; PICC, peripherally inserted central catheter; TCVC, tunneled central venous catheter.

Adapted with permission from Loveday HP et al. Epic 3: national evidence-based guidelines for preventing healthcare-associated infections in NHS hospitals in England. J Hosp Infect 2014;86(Suppl 1): S1-S70.

studies have shown that the incidence of CRI reduced with impregnated catheters, given their cost, they are best used in patients with high risk of CRI such as burn patients, neutropenic patients. ${ }^{2,12}$ It is useful in patients requiring a line for more than 5 days. ${ }^{2}$ Catheters made of polytetrafluroethylene and polyurethane have fewer CRIs when compared with polyethylene and polyvinylchloride. ${ }^{2,13}$-Table 2 highlights the various situations when a particular type of ICVAD is used.

\section{Patient Preparation}

Most catheter insertions can be undertaken under local anesthesia. However, if patients are nervous or a long procedural time is anticipated, sedation should be considered. In the case of children, not all cases will require sedation. Infants and toddlers will require sedation to ensure their cooperation. The authors have used feed and wrap technique without sedation for newborns. Older children and teenagers should be assessed on an individual basis.

Consensus guideline issued by the Society of Interventional Radiology and Cardiovascular and Interventional Radiological Society of Europe has classed the placement of PICC as a procedure with low risk of bleeding, thus recommending that international normalized ratio (INR) should be undertaken in those receiving warfarin or those with suspected

Table 2 ICVAD selection based on patient factors

\begin{tabular}{|l|l|l|}
\hline 1. & Thin and cachectic patient & Low-profile port \\
\hline 2. & Patient with obesity & High-profile port \\
\hline 3. & $\begin{array}{l}\text { Chemotherapy and } \\
\text { hyperalimentation }\end{array}$ & $\begin{array}{l}\text { Double-chamber } \\
\text { port }\end{array}$ \\
\hline 4. & Power injector compatible & Power port \\
\hline
\end{tabular}

Abbreviation: ICVAD, implanted central venous access device. liver disease, and activated partial thromboplastin time (aPTT) in patients receiving unfractionated heparin. Platelet count and hematocrit assessment are not recommended. TCVC and ICVAD placements are categorized as moderaterisk procedures. Routine INR and aPTT are recommended prior to the procedure. Platelet count and hematocrit assessment are not routinely recommended. ${ }^{14}$

In a personnel communication to one of the authors (V.R.), one center did not routinely undertake laboratory investigations. They were restricted to patients with preexisting hematological conditions or on medications that can affect the coagulation cascade. Though this is a single-center experience, more research is needed in this area. This may be of particular relevance to clinical practice in India, as this can help reduce unnecessary costs, which are borne by the patients.

As placement of CVCs is considered to be a clean procedure, routine administration of antibiotics is not recommended. However, antibiotic prophylaxis should be considered in immunocompromised patients who require a line placement. ${ }^{15}$

\section{Room and Table Preparation}

Interventional radiology suites are generally classed as clean procedural rooms. Most modern IR suites have laminar air flow system. The authors do not recommend that CVC placement be undertaken in areas where regular endoscopy and colonoscopy procedures are undertaken. Sometimes the procedural table is laid out prior to the patient's arrival to the angio suite. Even in these circumstances, the authors recommend that the nurse use maximum sterile barrier precautions when laying out the trolley.

Only the required personnel should be in the room. All of them should wear protective mask and cap, with the primary operator and the assistant using maximum sterile barrier precautions. This means wearing cap, mask, sterile gloves, and gown. In addition, full-body sterile drape should be used. $^{2}$ A long sterile cover should be used to cover the ultrasound probe and cable rather than a sterile glove or a smaller cover (-Fig. 1).

\section{Skin Antisepsis}

The commonly available agents are either chlorhexidine or povidone-based compounds. The recently published multicenter randomized CLEAN trial has shown that chlorhexidine-alcohol offers a much greater protection against shortterm CRIs than povidone iodine-alcohol combination. ${ }^{16}$ It also demonstrated that prior scrubbing had no effect on the infection rates. In this study, the concentration of chlorhexidine used was $2 \%$ in combination with $70 \%$ isopropyl alcohol.

Chlorhexidine-alcohol combination is more effective because of the immediate action of alcohol and subsequent residual activity of chlorhexidine. Povidone-iodine is rendered inactive by blood and other protein-rich biomaterials. $^{16}$ Recent evidence-based guidelines from the United States and the United Kingdom recommend the use of 
greater than $0.5 \%$ and $2 \%$ chlorhexidine with alcohol, respectively. ${ }^{2,13}$ Povidone-iodine in alcohol can be used in patients who are sensitive to chlorhexidine.

\section{Puncture Site}

When PICCs are placed by noninterventionalists or without ultrasound guidance, they tend to be placed in the antecubital fossa. However, both joint motion and muscular activity will lead to catheter motion, leading to trauma and endothelial damage and subsequent thrombosis and infection. Dawson proposed the PICC zone insertion method (ZIM). ${ }^{17}$ The arm is divided into three zones-yellow, green, and red-with the green zone being the ideal site for insertion (-Fig. 2).

The yellow zone or area of moisture corresponds to the upper one-third of the arm in which the moisture of the axilla increases the risk of infection. The red zone or zone of flexion refers to the lower one-third of the forearm in which there is increased catheter mobility making it prone to infection and thrombosis. The ideal needle insertion site is the upper part of the green zone (middle one-third of the arm).

The choice of vein tends to be in the following order: basilic vein, brachial veins, and cephalic vein. Though this is not absolute, the choice of vein should ensure low cathetervein ratio. This ensures that there is sufficient flow around the catheter to prevent sluggish flow and subsequent thrombosis. In their study, Sharp et al found that a catheter-to-vein ratio of $45 \%$ or less had a high sensitivity and specificity in reducing the risk of thrombosis. ${ }^{18}$ Ge et al found no significant differences in the infection and mechanical complication rates between patients with jugular or subclavian catheters. ${ }^{19}$ Femoral access should be avoided, particularly in patients with obesity, as there is a higher degree of infection and thrombosis rate. ${ }^{2,19}$

In the case of dialysis catheter, the right internal jugular vein is preferred as it offers a more direct route to the right atrium (RA). If possible, subclavian vein should be avoided as there is risk of central venous stenosis that can jeopardize the formation of upper limb fistulas. ${ }^{7}$ Femoral catheters are

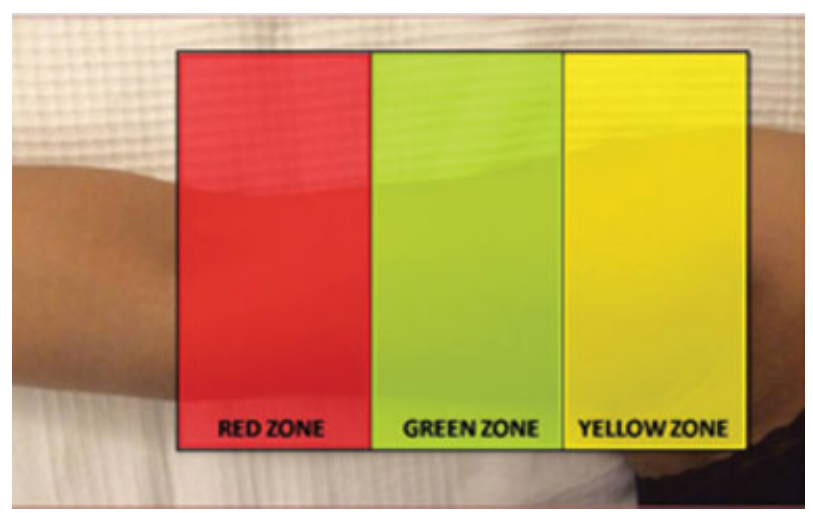

Fig. 2 The medial aspect of the arm, extending from the medial epicondyle to the axillary line is divided into three equal divisions, with the green zone being the ideal site for insertion. associated with a high risk of infection and should be considered as a last option. Femoral route for temporary dialysis catheter should be considered only in bed-bound patients and should not be in place for more than 1 week. ${ }^{7}$ Other access routes such as the translumbar, transhepatic, and chest wall collaterals can be used when conventional access points have been exhausted. They are associated with higher rates of infection and mechanical complications, necessitating their early removal or exchange. ${ }^{20}$

As a general rule, the preferred vein should not be on the dominant side. One should consider using distal veins first. If the central vein is occluded, an attempt can be made to recanalize the occluded vein. Alternatively, if the internal jugular vein is occluded, the catheter can be placed via an external jugular vein or enlarged collateral.

\section{Use of Ultrasound}

National Institute for Health and Care Excellence (NICE, United Kingdom) has issued guidance that in all elective cases ultrasound should be used during insertion of internal jugular vein catheter and it should be considered in most clinical scenarios, be it elective or emergency. ${ }^{21}$ This will obviate the mechanical complications that were observed in the Cochrane review undertaken by Ge et al. ${ }^{19}$

A recent Cochrane review found that the use of ultrasound for subclavian vein puncture reduced the risk of an arterial puncture and subsequent hematoma formation. However, there was no difference noted in the total complications, first-time success rates, the number of attempts, or the time taken to insert the catheter between ultrasound or landmark technique. For femoral vein punctures, first-attempt success rates were recorded with the use of ultrasound. However, there was no difference in complication rates, insertion time, arterial puncture, or hematoma formation between the two techniques. $^{22}$

\section{Catheter Tip Location}

It is important to ascertain the catheter tip position prior to use, particularly in catheters that have been placed at bedside. Unfortunately this is a subject of controversy. There is no accurate landmark that ascertains the distal superior vena cava (SVC) or SVC/RA junction on a chest radiograph. ${ }^{23}$ Inaccurate tip position is associated with increased risk of thrombosis due to vessel damage. It has been shown that the risk of thrombosis increases as the tip position changes from distal SVC/RA to the proximal SVC from 2.6 to $41.7 \%{ }^{24}$

Tip position noted at the end of the procedure will migrate when the position of the patient changes. When a PICC is placed in the abducted position, the tip demonstrates a caudal migration when the arm is adducted. Tip migration is also noted when the catheter is placed in the basilic vein and right arm. ${ }^{23}$ Cephalad catheter tip migration is noted in tunneled catheters when the patient moves from a supine to an upright position as abdominal contents descend leading to elongation of the veins and expansion of the RA (-Fig. 3 ). It has been reported that the average movement of the 
catheter tip is around $3 \mathrm{~cm} .{ }^{23}$ This is more pronounced in patients with obesity and in women with large breasts. Various recommendations to prevent tip migration include placing the tunnel more medially or parasternally and having separate tunnels for each of the external extremities of the catheter and a short subcutaneous tunnel when double-split catheters are used. ${ }^{25}$

A consensus statement for home parenteral nutrition by the American Society for Parenteral and Enteral Nutrition (ASPEN) states that the catheter tip be positioned near the junction of the SVA and RA and the tip should not point toward the SVC sidewall. ${ }^{26}$ Kidney Disease Outcomes Quality Initiative (KDOQI) guidelines recommend that the tip of temporary uncuffed catheters should be placed in the distal SVC, whereas the tip of tunneled catheters should be in the proximal RA. ${ }^{7}$ When femoral catheters are used, one must ensure that the tip reaches the RA or at least the inferior vena cava (IVC) so that flow rate of $300 \mathrm{~mL} / \mathrm{min}$ can be achieved. ${ }^{7,27}$ If a dual-lumen catheter with variable lengths are used, the distal tip should be placed in the RA and the proximal tip should lie $1 \mathrm{~cm}$ outside the RA. ${ }^{27}$

\section{Catheter Securement}

Catheters need to be secured to prevent migration and subsequent complications. Sutureless securement devices are generally recommended to reduce the incidence of infection. $^{13}$

Sutures have been traditionally used. They tend to be placed at the skin entry site and/or the catheter wing. The disadvantage of sutures is that they can erode through the skin, thus loosening the catheter and being a source of infection. ${ }^{28}$ In the case of cuffed tunneled catheters, the sutures are required for a period of 4 weeks, allowing for the cuff to integrate with the subcutaneous tissue.

Adhesive devices such as StatLock devices (Bard PV) can be used. Unfortunately these have to be changed every week, which adds to be cost. If left for long, there is disintegration of the adhesive and correct solvents may be required to remove it. $^{28}$ Subcutaneous securement devices such as SecurAcath

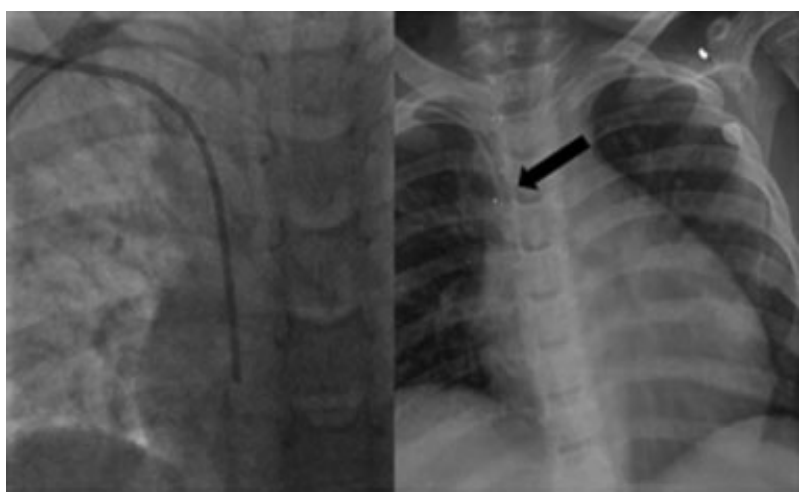

Fig. 3 The tip of the PICC line is in the right atrium on the supine projection. However, the tip position is in the superior vena cava (black arrow). Note the change in the shape of the cardiac silhouette from supine to erect imaging.
(Interrad Medical) prevent pistoning and side-to-side movement of the catheter. The anchor integrates with the tissue within 48 to 72 hours. ${ }^{28}$ While there is a learning curve associated with their use, it has led to decreased complication and easier dressing changes both for the patient and nursing staff. ${ }^{28,29}$

\section{Choice of Catheter Dressings}

If there is bleeding or ooze after insertion or the patient is diaphoretic, sterile gauze should be considered. The soiled dressing should be changed within 24 hours. If there are no such issues, transparent semipermeable dressings can be used. These provide the advantage of direct visualization of the insertion site. However, there is no proven advantage of one method over the other. 2,13

Newer antiseptic-impregnated dressings are available. Various studies have shown that they reduce the incidence of CRI. However, given the cost implications, they can be reserved for at-risk patients.

\section{Flushing Technique}

Rather than continuous flushing technique, pulsatile flushing technique should be used. In an in vitro experiment, it has been shown to have better clearance of microorganisms. ${ }^{30} \mathrm{In}$ a similar experimental model, Guiffant et al found that 10 intermittent flushes of $1-\mathrm{mL}$ boluses with an interval of 0.4 second between boluses were more efficient to rinse the catheter than shorter or longer pauses between two boluses. In spite of this, incomplete removal of the proteins was reported in their study. However, the clearance was better than continuous infusion. ${ }^{31}$ There are no studies quantifying the volume of normal saline needed for flushing of catheters. The general recommendation is that use of flushing volume should be twice that of the catheter and any add-on device. ${ }^{32}$ -Table 3 provides guidance on the recommended volumes for flushing of VAD.

\section{Catheter Replacement}

Routine catheter replacement is not recommended. There is no sufficient evidence to suggest that regular replacement will reduce the risk of infection. 2,13,33 Catheter replacement is recommended only if there are clinical signs of CRI. The presence of fever alone should not prompt the need to

Table 3 Frequency of catheter flushing

\begin{tabular}{|l|l|l|}
\hline 1. & Routine flushing every 7 d & $5-10 \mathrm{~mL}$ \\
\hline 2. & $\begin{array}{l}\text { After administration of viscous } \\
\text { products-blood products, parent- } \\
\text { eral nutrition, contrast media }\end{array}$ & $20 \mathrm{~mL}$ \\
\hline 3. & $\begin{array}{l}\text { After administration of nonviscous } \\
\text { products }\end{array}$ & $10 \mathrm{~mL}$ \\
\hline 4. & $\begin{array}{l}\text { After aspiration of blood for } \\
\text { sampling }\end{array}$ & $10 \mathrm{~mL}$ \\
\hline
\end{tabular}


change the catheter. Clinical acumen should be used to rule out other causes of fever, prior to attributing it to CRI. ${ }^{13}$ Over the wire exchange can be used if the catheter is being exchanged for mechanical reasons. If there is an infection, the catheter should be removed and a new puncture site has to be used. In addition, VAD should be removed at the earliest instance when the clinical requirement no longer exists.

\section{After-Care}

The eventual success of a line placement is not just in the technique. After-care of the catheter plays a very important role. Though this may not be the domain of an interventional radiologist, the authors feel that they can play an important role in facilitating it. In addition, interventional radiologists should be capable of managing the dysfunctional catheters such as blocked catheters, fibrin sheath formation, and fractured catheters. A detailed description of these techniques is beyond the scope of this article.

The end users of the catheters, that is, patients, nurses, and others who will be handling the catheter, should be trained. This ensures the increased longevity of the catheter and reduces various complications. It has been shown that active monitoring and intensive training reduce the infection rate by more than $65 \%{ }^{33}$ In hospital audit, process should be in place to track all the catheters that are placed, record any complications, and implement appropriate measures to reduce them.

\section{Conclusion}

Central venous catheters play a vital role in patient management. Selection of the appropriate catheter and appropriate care ensures better outcomes. During insertion, maximum sterile barrier precaution is recommended. The catheter with the least required lumens is selected and placed under ultrasound guidance. It is important to ensure the correct position of the tip to prevent vessel damage and subsequent thrombosis. Securement with sutureless devices reduces the risk of infections.

\section{Disclosures}

All the authors declare that they have nothing to disclose.

\section{References}

1 Griffiths V. Midline catheters: indications, complications and maintenance. Nurs Stand 2007;22(11):48-57, quiz 58

2 Loveday HP, Wilson JA, Pratt RJ, et al; UK Department of Health. epic3: national evidence-based guidelines for preventing healthcare-associated infections in NHS hospitals in England. J Hosp Infect 2014;86(Suppl 1):S1-S70

3 Bishop L, Dougherty L, Bodenham A, et al. Guidelines on the insertion and management of central venous access devices in adults. Int J Lab Hematol 2007;29(04):261-278

4 Chopra V, Flanders SA, Saint S, et al; Michigan Appropriateness Guide for Intravenouse Catheters (MAGIC) Panel. The Michigan Appropriateness Guide for Intravenous Catheters (MAGIC): results from a multispecialty panel using the RAND/UCLA Appropriateness Method. Ann Intern Med 2015;163(6, Suppl):S1-S40
5 Chopra V, Anand S, Hickner A, et al. Risk of venous thromboembolism associated with peripherally inserted central catheters: a systematic review and meta-analysis. Lancet 2013; 382(9889):311-325

6 CAVA-Cancer and Venous Access. DOI 10.1186/ISRCTN44504648. http://www.isrctn.com/ISRCTN44504648. Accessed April 19, 2017

7 Kinney R; Centers for Medicare and Medicaid Services. 2005 Annual Report: ESRD Clinical Performance Measures Project. Am J Kidney Dis 2006;48(04, Suppl 2):S1-S106

8 Ash SR. Advances in tunneled central venous catheters for dialysis: design and performance. Semin Dial 2008;21(06):504-515

9 O'Brien J, Paquet F, Lindsay R, Valenti D. Insertion of PICCs with minimum number of lumens reduces complications and costs. J Am Coll Radiol 2013;10(11):864-868

10 Johnston AJ, Streater CT, Noorani R, Crofts JL, Del Mundo AB, Parker RA. The effect of peripherally inserted central catheter (PICC) valve technology on catheter occlusion rates-the 'ELeCTRiC' study. J Vasc Access 2012;13(04):421-425

11 Pittiruti M, Emoli A, Porta P, Marche B, DeAngelis R, Scoppettuolo G. A prospective, randomized comparison of three different types of valved and non-valved peripherally inserted central catheters. J Vasc Access 2014;15(06):519-523

12 O'Grady NP, Alexander M, Burns LA, et al; Healthcare Infection Control Practices Advisory Committee (HICPAC). Guidelines for the prevention of intravascular catheter-related infections. Clin Infect Dis 2011;52(09):e162-e193

13 Miller DL, O'Grady NP; Society of Interventional Radiology. Guidelines for the prevention of intravascular catheter-related infections: recommendations relevant to interventional radiology for venous catheter placement and maintenance. J Vasc Interv Radiol 2012;23(08):997-1007

14 Patel IJ, Davidson JC, Nikolic B, et al; Standards of Practice Committee, with Cardiovascular and Interventional Radiological Society of Europe (CIRSE) Endorsement. Consensus guidelines for periprocedural management of coagulation status and hemostasis risk in percutaneous image-guided interventions. J Vasc Interv Radiol 2012;23(06):727-736

15 Venkatesan AM, Kundu S, Sacks D, et al; Society of Interventional Radiology Standards of Practice Committee. Practice guidelines for adult antibiotic prophylaxis during vascular and interventional radiology procedures. Written by the Standards of Practice Committee for the Society of Interventional Radiology and endorsed by the Cardiovascular Interventional Radiological Society of Europe and Canadian Interventional Radiology Association [corrected]. J Vasc Interv Radiol 2010;21(11):1611-1630, quiz 1631

16 Mimoz O, Lucet JC, Kerforne T, et al; CLEAN trial investigators. Skin antisepsis with chlorhexidine-alcohol versus povidone iodinealcohol, with and without skin scrubbing, for prevention of intravascular-catheter-related infection (CLEAN): an open-label, multicentre, randomised, controlled, two-by-two factorial trial. Lancet 2015;386(10008):2069-2077

17 Dawson RB. PICC zone insertion method (ZIM): a systematic approach to determine the ideal insertion site for PICCs in the upper arm. J Association Vasc Access 2011;16:162-165

18 Sharp R, Cummings M, Fielder A, Mikocka-Walus A, Grech C, Esterman $A$. The catheter to vein ratio and rates of symptomatic venous thromboembolism in patients with a peripherally inserted central catheter (PICC): a prospective cohort study. Int J Nurs Stud 2015;52(03):677-685

19 Ge X, Cavallazzi R, Li C, Pan SM, Wang YW, Wang FL. Central venous access sites for the prevention of venous thrombosis, stenosis and infection. Cochrane Database Syst Rev 2012; 14(03):CD004084

20 Pereira K, Osiason A, Salsamendi J. Vascular access for placement of tunneled dialysis catheters for hemodialysis: a systematic approach and clinical practice algorithm. J Clin Imaging Sci 2015;5:31 
21 Guidance on the use of ultrasound location device for placing central venous catheters. [Internet]. London, UK: National Institute for Health and Care Excellence; 2002 [updated 2014; cited 2016 Jan 6]. https://www.nice.org.uk/guidance/ta49

22 Brass P, Hellmich M, Kolodziej L, Schick G, Smith AF. Ultrasound guidance versus anatomical landmarks for subclavian or femoral vein catheterization. Cochrane Database Syst Rev 2015;1: CD011447

23 Vesely TM. Central venous catheter tip position: a continuing controversy. J Vasc Interv Radiol 2003;14(05):527-534

24 Cadman A, Lawrance JA, Fitzsimmons L, Spencer-Shaw A, Swindell $R$. To clot or not to clot? That is the question in central venous catheters. Clin Radiol 2004;59(04):349-355

25 Celebi-Onder S, Schmidt RJ, Holley JL. Treating the obese dialysis patient: challenges and paradoxes. Semin Dial 2012;25(03):311-319

26 Steiger E; HPEN Working Group. Consensus statements regarding optimal management of home parenteral nutrition (HPN) access. JPEN J Parenter Enteral Nutr 2006;30(1, Suppl):S94-S95

27 Santoro D, Benedetto F, Mondello P, et al. Vascular access for hemodialysis: current perspectives. Int J Nephrol Renovasc Dis 2014;7:281-294
28 Macklin D, Blackburn PL. Central venous catheter securement: using the healthcare and technology synergy model to take a closer look. The J Association Vascular 2015;20:45-50

29 Elen Hughes M. Reducing PICC migrations and improving patient outcomes. Br J Nurs 2014;23(02):S12-S8, S14-S18

30 Ferroni A, Gaudin F, Guiffant G, et al. Pulsative flushing as a strategy to prevent bacterial colonization of vascular access devices. Med Devices (Auckl) 2014;7:379-383

31 Guiffant G, Durussel JJ, Merckx J, Flaud P, Vigier JP, Mousset P. Flushing of intravascular access devices (IVADs)-efficacy of pulsed and continuous infusions. J Vasc Access 2012;13(01): 75-78

32 Goossens GA. Flushing and locking of venous catheters: available evidence and evidence deficit. Nurs Res Pract 2015;2015:985686

33 Hentrich M, Schalk E, Schmidt-Hieber M, et al; Infectious Diseases Working Party of the German Society of Hematology and Medical Oncology. Central venous catheter-related infections in hematology and oncology: 2012 updated guidelines on diagnosis, management and prevention by the Infectious Diseases Working Party of the German Society of Hematology and Medical Oncology. Ann Oncol 2014;25(05):936-947 